PDES, SUBMANIFOLDS AND

AFFINE DIFFERENTIAL GEOMETRY

BANACH CENTER PUBLICATIONS, VOLUME 57

INSTITUTE OF MATHEMATICS

POLISH ACADEMY OF SCIENCES

WARSZAWA 2002

\title{
SURFACES WITH PRESCRIBED WEINGARTEN OPERATOR
}

\author{
UDO SIMON \\ Fak. II, Institut Mathematik, MA 8-3, TU-Berlin, D-10623 Berlin, Germany \\ E-mail: simon@math.tu-berlin.de \\ KONRAD VOSS \\ Department of Mathematics, ETH-Zentrum, CH-8092 Zürich, Switzerland \\ E-mail:voss@math.ethz.ch \\ LUC VRANCKEN \\ Laboratoire de Mathématiques, LAMATH, ISTV2, Université de Valenciennes \\ 59313 Valenciennes Cedex 9, France \\ E-mail: luc.vrancken@web.de \\ MARTIN WIEHE \\ Fak. II, Institut Mathematik, MA 8-3, TU-Berlin, D-10623 Berlin, Germany \\ E-mail:wiehe@math.tu-berlin.de
}

\begin{abstract}
We investigate pairs of surfaces in Euclidean 3-space with the same Weingarten operator in case that one surface is given as surface of revolution. Our local and global results complement global results on ovaloids of revolution from [S-V-W-W].
\end{abstract}

Introduction. In $[\mathrm{S}-\mathrm{V}-\mathrm{W}-\mathrm{W}]$ we studied global uniqueness results of the following type:

TheOrem A. Let $x, x^{\#}: M \rightarrow E^{3}$ be ovaloids in Euclidean 3-space with nowhere dense umbilics and with the property that, at any $p \in M$, the Weingarten operators $S, S^{\#}$

2000 Mathematics Subject Classification: 53A05, 53C24, 53C40, 53C42.

Key words and phrases: Weingarten operator, surfaces of revolution.

Research of U. Simon and M. Wiehe partially supported by DFG. Both authors thank the Deutsch-Polnische Stiftung for partial support for the conference at the Banach Center in September 2000.

Research of L. Vrancken supported by a research fellowship of the Alexander von Humboldt Stiftung (Germany).

The paper is in final form and no version of it will be published elsewhere. 
and the spherical volume forms $\omega(\mathrm{III}), \omega\left(\mathrm{II}^{\#}\right)$ coincide:

$$
S=S^{\#}, \quad \omega(\text { III })=\omega\left(\text { III }^{\#}\right) .
$$

Then $x, x^{\#}$ are congruent.

Corollary B. Let $x, x^{\#}: M \rightarrow E^{3}$ be ovaloids such that $S=S^{\#}$ and $\omega(\mathrm{III})=\omega\left(\mathrm{II}^{\#}\right)$. If $x$ is analytic then $x, x^{\#}$ are congruent up to a reparametrization.

The basic idea for the proof of Theorem A is to consider the unique, I-selfadjoint, positive definite operator $\mathrm{L}$ defined by

$$
\mathrm{I}^{\#}(v, w)=: \mathrm{I}(L v, L w)
$$

and to study its algebraic and analytic properties. A second tool is to use the Codazzi equations for $S=S^{\#}$ in terms of the two Levi-Civita connections $\nabla=\nabla(\mathrm{I})$ and $\nabla^{\#}=$ $\nabla\left(\mathrm{I}^{\#}\right)$ to get relations for the symmetric $(1,2)$ difference tensor $\left(\nabla-\nabla^{\#}\right)$ between the connections which finally lead to PDEs for the operator $L$.

If one follows the proof it seems that one might drop the assumption on the volume forms. We would like to state the following

Conjecture. Let $x, x^{\#}: M \rightarrow E^{3}$ be ovaloids with nowhere dense umbilics and with $S=S^{\#}$ at corresponding points. Then $x, x^{\#}$ are congruent.

An affirmative answer to this conjecture would be an extrinsic counterpart to the intrinsic rigidity result of Cohn-Vossen [COHN-V] which states that two isometric ovaloids in $E^{3}$ are congruent. In Section 4 of $[\mathrm{S}-\mathrm{V}-\mathrm{W}-\mathrm{W}]$ we gave the following partial answer to our conjecture.

Theorem C. Let $x: M \rightarrow E^{3}$ be an ovaloid of revolution with nowhere dense umbilics and let $x^{\#}: M \rightarrow E^{3}$ be another ovaloid with $S=S^{\#}$ at corresponding points. Then $x, x^{\#}$ are congruent.

At the end of $[\mathrm{S}-\mathrm{V}-\mathrm{W}-\mathrm{W}]$ we showed by an explicit example that there exists a nontrivial 1-parameter family of complete surfaces of revolution $x_{c}: M \rightarrow E^{3}$ having the same Weingarten operator. It is the aim of this paper (Sections 2-4) to give a complete discussion of the local situation of a pair of surfaces $x, x^{\#}: M \rightarrow E^{3}$, where $x$ is a surface of revolution and where the Weingarten operators coincide for any $p \in M$. In Section 5 we state the local results, in Section 6 we extend Theorem C.

2. Surfaces of revolution. We summarize well known properties of surfaces of revolution which we will need for our discussion.

Consider a surface of revolution given in terms of parameters $\left(u^{1}, u^{2}\right)$ by

$$
x\left(u^{1}, u^{2}\right)=\left(r\left(u^{1}\right) \cos u^{2}, r\left(u^{1}\right) \sin u^{2}, s\left(u^{1}\right)\right), \quad r \geq 0,
$$

where $u^{1}$ parametrizes the meridians as arc length parameter and $u^{2}$ parametrizes the parallels of latitude with radius $r\left(u^{1}\right)$ and $r$ and $s$ are differentiable functions. For a function $f=f\left(u^{1}\right)$ we write $f^{\prime}:=d f / d u^{1}$; thus we have

$$
r^{\prime}\left(u^{1}\right)^{2}+s^{\prime}\left(u^{1}\right)^{2}=1 .
$$


$u^{1}, u^{2}$ are curvature line parameters for all points with $r\left(u^{1}\right)>0$. In case that the functions $r\left(u^{1}\right)>0, s\left(u^{1}\right)$ are defined for $0<u^{1}<\Lambda$ and also for $u^{1}=0$ or $u^{1}=\Lambda$ such that

$$
r(0)=0, r^{\prime}(0)=1 \quad \text { or } \quad r(\Lambda)=0, r^{\prime}(\Lambda)=-1,
$$

we call such points "poles" $P_{S}$ or $P_{N}\left(u^{1}=0\right.$ or $\left.u^{1}=\Lambda\right)$; for symmetry reasons they are umbilics. Near a pole, $x^{1}=r\left(u^{1}\right) \cos u^{2}, x^{2}=r\left(u^{1}\right) \sin u^{2}$ are parameters for the surface. In general, the parameter $u^{2}$ will be taken modulo $2 \pi$, i.e. $u^{2} \in S^{1}$; but there will be cases where one has to pass to a covering surface by taking $u^{2} \in \mathbb{R}$. In every case, according to the domain of the parameters, there is a manifold $M$ of dimension two such that (2.0.1) defines an immersion $x: M \rightarrow E^{3}$.

It follows by a straightforward computation that the first fundamental form $\mathrm{I}=: g$ has the representation on $M \backslash\left\{P_{N}, P_{S}\right\}$ :

$$
g_{11}=1, g_{12}=0, g_{22}=r^{2},
$$

and $r$ and $s$ satisfy

$$
S \partial_{1}=\left(r^{\prime} s^{\prime \prime}-r^{\prime \prime} s^{\prime}\right) \partial_{1} \quad \text { and } \quad S \partial_{2}=\frac{s^{\prime}}{r} \partial_{2},
$$

where $\left\{\partial_{1}, \partial_{2}\right\}$ denotes the Gauß basis associated to the local parameters.

The equation $\left(r^{\prime}\right)^{2}+\left(s^{\prime}\right)^{2}=1$ suggests introducing the function $\sigma=\sigma\left(u^{1}\right)$ by $\cos \sigma:=$ $r^{\prime}$ and $\sin \sigma:=s^{\prime}$. The Weingarten operator is represented by the matrix

$$
S:\left(\begin{array}{cc}
k_{1} & 0 \\
0 & k_{2}
\end{array}\right)=\left(\begin{array}{cc}
\sigma^{\prime} & 0 \\
0 & \frac{1}{r} \sin \sigma
\end{array}\right)
$$

with $k_{1}, k_{2}$ as principal curvatures. The Codazzi equations reduce to the equation

$$
k_{2}^{\prime}=\frac{r^{\prime}}{r}\left(k_{1}-k_{2}\right) \text {, }
$$

while the Gauß integrability condition reads

$$
r^{\prime \prime}+K r=0
$$

3. Pairs of surfaces with the same Weingarten operator. At the beginning of this section we recall some local results from $[\mathrm{S}-\mathrm{V}-\mathrm{W}-\mathrm{W}]$. For a moment, let $M$ be a connected, oriented $C^{\infty}$-manifold of dimension two; later again, we will restrict to $M$ as given in Section 2. Let $x, x^{\#}: M \rightarrow E^{3}$ be a pair of surfaces with first fundamental forms $g, g^{\#}$ and associated Levi-Civita connections $\nabla:=\nabla(g), \nabla^{\#}:=\nabla\left(g^{\#}\right)$ and Weingarten operators $S=S^{\#}$. We have the following facts from section 2 in $[\mathrm{S}-\mathrm{V}-\mathrm{W}-\mathrm{W}]$.

\subsection{FACTS.}

(i) There exists a unique g-self-adjoint, positive definite operator $L$ such that

$$
g^{\#}(u, v)=g(L u, L v)
$$

for tangent vectors $u, v$. Denote the positive eigenvalue functions of $L$ by $\lambda_{1}, \lambda_{2}$; they are continuous on $M$ and, if $\lambda_{1} \neq \lambda_{2}$, differentiable.

(ii) The operator $L$ and the Weingarten operator $S=S^{\#}$ commute. 
(iii) If $x, x^{\#}$ admit a common curvature line parametrization on an open set $U \subset M$, then the eigendirections of $S$ are eigendirections of $L$, and we have the following local matrix representations:

$$
\begin{aligned}
& g:\left(\begin{array}{cc}
g_{11} & 0 \\
0 & g_{22}
\end{array}\right), \quad g^{\#}:\left(\begin{array}{cc}
\lambda_{1}^{2} g_{11} & 0 \\
0 & \lambda_{2}^{2} g_{22}
\end{array}\right), \\
& L:\left(\begin{array}{cc}
\lambda_{1} & 0 \\
0 & \lambda_{2}
\end{array}\right), \quad S=S^{\#}:\left(\begin{array}{cc}
k_{1} & 0 \\
0 & k_{2}
\end{array}\right) .
\end{aligned}
$$

(iv) If $\lambda_{1}, \lambda_{2}$ are differentiable, their partial derivatives in terms of curvature line parameters satisfy

$$
\partial_{2} \lambda_{1}=0=\partial_{1} \lambda_{2}
$$

These equations are consequences of the Codazzi equations for $S=S^{\#}$ in terms of $\nabla$ and $\nabla^{\#}$, resp.; they are an essential tool for our discussion below.

3.2. Codazzi equations. In curvature line parameters and with associated Gauß basis $\left\{\partial_{1}, \partial_{2}\right\}$, the Codazzi equations for $x$ read:

$$
\begin{aligned}
& 2 \partial_{2} k_{1}=-\partial_{2} \log \left(g_{11}\right)\left(k_{1}-k_{2}\right), \\
& 2 \partial_{1} k_{2}=\partial_{1} \log \left(g_{22}\right)\left(k_{1}-k_{2}\right) .
\end{aligned}
$$

4. The local discussion. Let $x: M \rightarrow E^{3}$ be a surface of revolution as given in Section 2 and $x^{\#}: M \rightarrow E^{3}$ a second surface with the same parameter domain $M$. Let $U \subset M$ be open, connected and assume that $x$ is without umbilics on $U$. Then we can apply the parametrization and matrix representations from 3.1(iii); moreover, $\lambda_{1}$ and $\lambda_{2}$ are differentiable on $U$.

We discuss the consequences of the integrability conditions in terms of the representations in 3.1(iii). One easily verifies that there is no further information from the Codazzi equations. The Gauß equation and $K=\operatorname{det} S=\operatorname{det} S^{\#}=K^{\#}$ lead to an ODE which is crucial for our discussion; see Section 4 in $[\mathrm{S}-\mathrm{V}-\mathrm{W}-\mathrm{W}]$.

4.1. Proposition. The functions $r=r\left(u^{1}\right)$ and $\tau:=\left(\lambda_{1}\right)^{-2}-1=\tau\left(u^{1}\right)$ satisfy

$$
r^{\prime \prime} \tau+\frac{1}{2} r^{\prime} \tau^{\prime}=0
$$

thus there exists $c \in \mathbb{R}$ such that

$$
\left(r^{\prime}\right)^{2} \tau=c
$$

\subsection{Consequences.}

(i) If $c=0$, then $r=$ const or $\lambda_{1}=1$.

(ii) If $c \neq 0$, then $r^{\prime} \neq 0$ and

$$
\lambda_{1}=\frac{\left|r^{\prime}\right|}{\sqrt{\left(r^{\prime}\right)^{2}+c}} \neq 1 .
$$

4.3. Discussion of the case $c=0$.

(a) If $r=: r_{0}=$ const then $\left(r^{\prime}\right)^{2}+\left(s^{\prime}\right)^{2}=1$ implies $s=u^{1}$ and $x$ lies on a circular cylinder

$$
x=\left(r_{0} \cos u^{2}, r_{0} \sin u^{2}, u^{1}\right) .
$$


Choosing arbitrary positive functions $\lambda_{1}\left(u^{1}\right), \lambda_{2}\left(u^{2}\right)$, the invariants $g^{\#}$ and $S^{\#}$ are given by 3.1 (iii); thus the surface $x^{\#}$ is uniquely determined.

Reparametrize

$$
\bar{u}^{1}:=\int^{u^{1}} \lambda_{1}(u) d u, \quad \bar{u}^{2}:=\int^{u^{2}} \lambda_{2}(v) d v .
$$

One verifies that the solution $x^{\#}$ is given by

$$
x^{\#}=\left(r_{0} \cos \bar{u}^{2}, r_{0} \sin \bar{u}^{2}, \bar{u}^{1}\right),
$$

thus $x^{\#}$ is another parametrization of the same circular cylinder and the diffeomorphism $\left(u^{1}, u^{2}\right) \mapsto\left(\bar{u}^{1}, \bar{u}^{2}\right)$ preserves the Weingarten operator: $S=S^{\#}$.

(b) If $\lambda_{1}=1$ we again reparametrize, using (4.3.1). Analogously to the foregoing we arrive at

$$
x^{\#}=\left(r\left(u^{1}\right) \cos \bar{u}^{2}, r\left(u^{1}\right) \sin \left(\bar{u}^{2}\right), s\left(u^{1}\right)\right) .
$$

$x^{\#}$ is a modified parametrization of the same surface of revolution. Again the diffeomorphism $\left(u^{1}, u^{2}\right) \mapsto\left(u^{1}, \bar{u}^{2}\right)$ preserves the Weingarten operator.

4.4. Discussion of the case $c \neq 0$. For fixed $c$, the function $\lambda_{1}$ is given by $4.2(\mathrm{ii})$; we reparametrize $x_{c}^{\#}$ by

$$
\bar{u}_{c}^{1}:=\int^{u^{1}} \lambda_{1}(u) d u, \quad \bar{u}_{c}^{2}:=a \int^{u^{2}} \lambda_{2}(v) d v, \quad 0<\sqrt{1+c}=: a \in \mathbb{R} .
$$

Introduce the functions

$$
\bar{r}\left(\bar{u}_{c}^{1}\right):=\frac{1}{a} r\left(u^{1}\right) \text { and } \bar{s}\left(\bar{u}^{1}\right):=\frac{1}{a} \int^{u^{1}} \sqrt{\left(a \lambda_{1}\right)^{2}-\left(r^{\prime}\right)^{2}} d u .
$$

Choosing the constant $a$ as given in (4.4.1) and using 4.2(ii), we get:

4.5. Proposition. In terms of the coordinates $\left(\bar{u}_{c}^{1}, \bar{u}_{c}^{2}\right)$, which both depend on the constant $c$, the surface $x_{c}^{\#}$ is given by

$$
x_{c}^{\#}\left(\bar{u}^{1}, \bar{u}^{2}\right)=\left(\bar{r}\left(\bar{u}^{1}\right) \cos \bar{u}^{2}, \bar{r}\left(\bar{u}^{1}\right) \sin \bar{u}^{2}, \bar{s}\left(\bar{u}^{1}\right)\right) ;
$$

in particular, $x_{c}^{\#}$ is a surface of revolution; moreover $S=S_{c}^{\#}$ in corresponding points $\left(u^{1}, u^{2}\right) \mapsto\left(\bar{u}_{c}^{1}, \bar{u}_{c}^{2}\right)$ for any $c \neq 0 . x_{c}^{\#}$ is also defined for $c=0$; the choice $\lambda_{2}=a^{-1}$ (i.e. $\left.\bar{u}^{2}=u^{2}\right)$ yields $x_{0}^{\#}=x$.

4.6. Remark. (a) Recall $\left(r^{\prime}\right)^{2} \leq 1$ from (2.0.2). According to 4.2(ii) the inequality $c \neq 0$ implies $\lambda_{1} \neq 1$, in particular:

(i) $c>0 \Leftrightarrow \lambda_{1}<1$; in this case we have $\lambda_{1} \leq \frac{1}{\sqrt{1+c}}$;

(ii) $c<0 \Leftrightarrow \lambda_{1}>1$; in this case we have $-1<c$ and $\lambda_{1} \geq \frac{1}{\sqrt{1+c}}$.

In both cases we have $\lambda_{1} \neq \sqrt{1+c}$.

(b) We discuss the special case $c \neq 0, \lambda_{1}=$ const $\neq 1$ and $\lambda_{1} \lambda_{2}=1$ for (4.1.1). Integrating (4.1.1), we get $r=\lambda_{1} \sqrt{c\left(1-\lambda_{1}^{2}\right)^{-1}} u^{1}$ and $s=\sqrt{\left(1-\lambda_{1}^{2}\right)^{-1}\left\{1-(1+c) \lambda_{1}^{2}\right\}} u^{1}$ from (2.0.2); thus we have a two-parameter family of circular cones

$$
x_{c, \lambda_{1}}=u^{1} \sqrt{\frac{c}{1-\lambda_{1}^{2}}}\left(\lambda_{1} \cos u^{2}, \lambda_{1} \sin u^{2}, \sqrt{\frac{1-(1+c) \lambda_{1}^{2}}{c}}\right) .
$$


For fixed $c, \lambda_{1}, \lambda_{2}=\lambda_{1}^{-1},(4.4 .1)$ and (4.4.2) yield the corresponding family of cones

$$
x_{c, \lambda_{1}}^{\#}=\lambda_{1} u^{1} \sqrt{\frac{c}{(1+c)\left(1-\lambda_{1}^{2}\right)}}\left(\cos \bar{u}^{2}, \sin \bar{u}^{2}, \sqrt{\frac{1-(1+c) \lambda_{1}^{2}}{c}}\right)
$$

having the same Weingarten operator and the same Riemannian volume form at $\left(u^{1}, u^{2}\right)$, but being non-isometric (and thus non-congruent). Especially $\frac{s}{r} \neq \frac{s^{\#}}{r^{\#}}$ and $\bar{u}^{2}=\frac{\sqrt{1+c}}{\lambda_{1}} u^{1} \neq$ $u^{1}$ since $\lambda_{1} \neq \sqrt{1+c}$.

(c) In $[\mathrm{S}-\mathrm{V}-\mathrm{W}-\mathrm{W}]$ we illustrated Proposition 4.5 by the example of the elliptic paraboloid

$$
x(u, v)=\left(u \cos v, u \sin v, \frac{1}{2} u^{2}\right)
$$

having the same Weingarten operator as any surface of the one-parameter family of strongly convex surfaces of revolution

$$
x_{c}(u, v)=\left(\frac{u}{\sqrt{1+c}} \cos v, \frac{u}{\sqrt{1+c}} \sin v, \frac{1}{c}\left[\sqrt{1+c \frac{u^{2}}{1+c}}-1\right]\right) .
$$

For the curve $m: u \mapsto(r(u), s(u))$, the equation $c^{2}\left(s+\frac{1}{c}\right)^{2}-c r^{2}=1$ holds: In case $c>0, m$ is the part of a hyperbola given by $s>0$ and the surfaces $x_{c}$ are complete. For $-1<c<0, x_{c}$ is half an ellipsoid.

5. Local results. In this section we state a series of consequences of the local discussion in Section 4. As before, $x: M \rightarrow E^{3}$ is a surface of revolution with the representation

$$
x\left(u^{1}, u^{2}\right)=\left(r\left(u^{1}\right) \cos u^{2}, r\left(u^{1}\right) \sin u^{2}, s\left(u^{1}\right)\right)
$$

on $M$; we assume that the umbilics are nowhere dense. Moreover, let $x^{\#}: M \rightarrow E^{3}$ be a surface with $S^{\#}=S$.

The detailed discussion in Section 4 admits the following implications.

5.1. Let $x, x^{\#}: M \rightarrow E^{3}$ be given as before; then there exist coordinates $\bar{u}^{1}, \bar{u}^{2}$ in $M$, and differentiable functions $\bar{r}=\bar{r}\left(\bar{u}^{1}\right), \bar{s}=\bar{s}\left(\bar{u}^{1}\right)$, such that $x^{\#}$ has the representation (modulo congruences in $E^{3}$ ):

$$
x^{\#}\left(\bar{u}^{1}, \bar{u}^{2}\right)=\left(\bar{r}\left(\bar{u}^{1}\right) \cos \bar{u}^{2}, \bar{r}\left(\bar{u}^{1}\right) \sin \bar{u}^{2}, \bar{s}\left(\bar{u}^{1}\right)\right) ;
$$

in particular, $x^{\#}$ again is a surface of revolution.

Proof. Sections 4.2 - 4.6 give a discussion of all possible cases.

5.2. There exists a non-trivial one-parameter family of strongly convex surfaces of revolution $x_{c}: M \rightarrow E^{3}$ having the same Weingarten operator at corresponding points. For $c>0$, the examples in 4.6(c) are complete and admit a bijective orthogonal mapping onto the plane $\left(x^{1}, x^{2}, 0\right) \subset \mathbb{R}^{3}$.

5.3. There exist non-isometric two-parameter families $x=x_{c, \lambda_{1}}, x^{\#}=x_{c, \lambda_{1}}^{\#}$ of surfaces of revolution (see 4.6(b)) with the properties

(i) the surfaces $x, x^{\#}$, have the same Weingarten operator and nowhere dense umbilics;

(ii) the surfaces $x, x^{\#}$ have the same Riemannian volume.

Such surfaces necessarily are circular cones. 
Proof. Almost everywhere we can introduce the local parameters from 3.1. (ii) implies $1=\operatorname{det} L=\lambda_{1} \cdot \lambda_{2}$ which together with $\partial_{2} \lambda_{1}=0=\partial_{1} \lambda_{2}$ gives $\lambda_{1}=$ const $\neq 1, \lambda_{2}=$ const $\neq 1$ (as $x, x^{\#}$ are assumed to be non-isometric). Then $c \neq 0$ in (4.1.1), and 4.6(b) describes the solution.

REMARK. The foregoing result in particular implies that both metrics $g, g^{\#}$ must be flat. Thus any pair $x, x^{\#}: M \rightarrow E^{3}$, where $x$ is a non-flat surface of revolution with nowhere dense umbilics which satisfies (i) and (ii) in 5.3, must be congruent.

5.4. Let $x, x^{\#}$ be given as in the beginning of this section. Let $p \in M$ be a pole for $x$. Then $p$ is also a pole for the surface of revolution $x^{\#}$. Using 3.1(i), we have $\lambda_{1}=\lambda_{2}$ at $p$.

Proof. 4.3(b) or 4.4; other cases are excluded.

5.5. Corollary. Let $D \subset M$ be a geodesic disc for $x$ (open or closed) with the pole $p$ as center; then $\lambda_{2}=$ const on $D$.

Proof. See the proof of Theorem 4.1 in $[\mathrm{S}-\mathrm{V}-\mathrm{W}-\mathrm{W}]$.

5.6. Corollary. Assume $r \neq$ const and let the disc $D$ from Corollary 5.5 contain a point (and then a parallel of latitude) with the property $r^{\prime}(p)=0\left(\right.$ or $\left.\lim _{q \rightarrow p} r^{\prime}(q)=0\right)$. Then $x$ and $x^{\#}$ are congruent on $D$.

Proof. Proposition 4.1 and Corollary 5.5 imply $1=\lambda_{1}=\lambda_{2}$ and thus $g=g^{\#}$.

6. Global results. In Section 4 of $[\mathrm{S}-\mathrm{V}-\mathrm{W}-\mathrm{W}]$ we considered ovaloids of revolution $x, x^{\#}: M \rightarrow E^{3}$ with nowhere dense umbilics and $S=S^{\#}$. We proved that $x, x^{\#}$ must be congruent (see Theorem $\mathrm{C}$ ). The comments in 5.2 show that, for a rigidity result, one cannot weaken the assumptions and consider $x$ to be a complete convex surface of revolution instead. Nevertheless, we can generalize Theorem $\mathrm{C}$ as follows.

6.1. TheOREM. Let $M$ be a surface of genus zero and $x, x^{\#}: M \rightarrow E^{3}$ be immersions. As before assume that $x$ is a surface of revolution as given in (2.0.1) having nowhere dense umbilics and $r\left(u^{1}\right) \neq$ const on open nonempty sets. Then $S=S^{\#}$ implies the congruence of $x$ and $x^{\#}$.

Proof. $\operatorname{Genus}(M)=0$ implies that the curve $u^{1} \mapsto\left(r\left(u^{1}\right), s\left(u^{1}\right)\right)$ is an arc with $r\left(u^{1}\right)>0$ for $0<u^{1}<\Lambda$ and $r(0)=r(\Lambda)=0$; thus $r^{\prime}$ has a zero between 0 and $\Lambda$, and Corollaries 5.5 and 5.6 imply $\lambda_{1}=\lambda_{2}=1$ on $M$.

\section{References}

[COHN-V] S. Cohn-Vossen, Zwei Sätze über die Starrheit der Eiflächen, Nachr. der Ges. der Wissensch. zu Göttingen, Math.-Phys. Kl., Jahrg. 1927, 125-134.

[S-V-W-W] U. Simon, L. Vrancken, C. P. Wang and M. Wiehe, Intrinsic and extrinsic geometry of ovaloids and rigidity, in: Geometry and Topology of Submanifolds X, World Scientific, Singapore, 2000, 284-293. 
Addendum (August 30, 2001). We learned from discussions with R. Bryant and E. V. Ferapontov about classical local results (due to E. Cartan, S. P. Finnikoff and B. Gambier) on surfaces with prescribed Weingarten (shape) operator; E. V. Ferapontov recently summarized such results in case the Weingarten operator has maximal rank. He also studied relations of the topic with hydrodynamics. Below we add a list of references which additionally contains two very recent papers on this topic by R. Bryant and K. Voss. The papers listed below, together with our above results, essentially give a complete local treatment of surfaces with prescribed Weingarten (shape) operator.
R. L. Bryant,
É. Cartan,
E. V. Ferapontov,
E. V. Ferapontov,
E. V. Ferapontov,

S. P. Finikoff,

K. Voss,
On surfaces with prescribed shape operator, Results Math. 40 (2001), to appear.

Les systèmes différentiels extérieurs et leurs applications géométriques, Actualités scient. et ind. 994, Hermann, Paris, 1945.

Hamiltonian systems of hydrodynamic type and their realization on hypersurfaces of a pseudoeuclidean space, Geom. Sbornik, VINITI, 22 (1990) 59-96 (Soviet J. Math. 55 (1991) 1970-1995). Compatible Poisson brackets of hydrodynamic type, J. Phys. A: Math. Gen. 34 (2001), 2377-2388. (arXiv:math.DG/0005221) Surfaces in 3-space possessing nontrivial deformations preserving the shape operator, in: Proc. conf. "Integrable systems in Differential Geometry", Tokyo, July 2000, to appear.

Surfaces dont les lignes de courbure se correspondent avec égalité des rayons de courbure principaux homologues, C. R. Acad. Sci. Paris 197 (1933), 984-986.

S. P. Finikoff et B. Gambier, Surfaces dont les lignes de courbure se correspondent avec égalité des rayons de courbure principaux, Ann. Sci. Ecole Norm. Sup. (3) 50 (1933), 319-370.

On the shape operator of surfaces in space forms, Results Math. 40 (2001), to appear. 